ALEA, Lat. Am. J. Probab. Math. Stat. 17, 589-607 (2020)

DOI: $10.30757 /$ ALEA.v17-23

\title{
F-KPP Scaling limit and selection principle for a Brunet-Derrida type particle system
}

\author{
Pablo Groisman, Matthieu Jonckheere and Julián Martínez \\ Departamento de Matemática, FCEN, Universidad de Buenos Aires, \\ IMAS-CONICET and NYU-ECNU Institute of Mathematical Sciences at \\ NYU Shanghai \\ E-mail address: pgroisma@dm.uba.ar \\ Instituto de Cálculo, FCEN, Universidad de Buenos Aires and CONICET \\ E-mail address: mjonckhe@dm.uba.ar \\ Departamento de Matemática, FIUBA, Universidad de Buenos Aires, \\ Instituto de Cálculo-CONICET, FCEN \\ E-mail address: jfmartinez@fiuba.uba.ar
}

Abstract. We study a particle system with the following diffusion-branchingselection mechanism. Particles perform independent one dimensional Brownian motions and on top of that, at a constant rate, a pair of particles is chosen uniformly at random and both particles adopt the position of the rightmost one among them. We show that the cumulative distribution function of the empirical measure converges to a solution of the Fisher-Kolmogorov-Petrovskii-Piskunov (F-KPP) equation and use this fact to prove that the system selects the minimal macroscopic speed as the number of particles goes to infinity.

\section{Introduction}

The F-KPP equation

$$
\begin{aligned}
& \partial_{t} u=\frac{1}{2} \partial_{x}^{2} u+u^{2}-u, \quad x \in \mathbb{R}, t>0, \\
& u(0, x)=u_{0}(x), \quad x \in \mathbb{R},
\end{aligned}
$$

was first introduced as a central model of front propagation for reaction-diffusion phenomena. Both Fisher and Kolmogorov, Petrovskii and Piskunov (Fisher, 1937; Kolmogorov et al., 1937) independently proved the existence of an infinite number of traveling wave solutions.

Received by the editors April 4th, 2019; accepted April 20th, 2020.

2010 Mathematics Subject Classification. 60K35, 60J25, 60B12.

Key words and phrases. Branching-selection, particle systems, velocity, F-KPP equation.

This research was supported in part by grants UBACYT 20020160100147BA and PICT 20153154 . 
In 1975, McKean established a first link with a microscopic model (a particle system), showing an exact connection with Branching Brownian motion (BBM) McKean (1975). This sprouted a large effort of research focusing on defining corresponding microscopic models for front propagation both in the physics and mathematics literature. The seminal papers Brunet and Derrida $(1997,2001)$ set the basic questions on the influence of microscopic effects on the front propagation properties. Three types of models were then considered: adding noise to the deterministic equation (Brunet et al., 2006; Mueller et al., 2008, 2011), introducing a cut-off (Benguria and Depassier, 2007; Dumortier et al., 2007) or defining truly microscopic models via interacting particles systems conserving the total number of particles (Brunet et al., 2007; Durrett and Remenik, 2011; Maillard, 2016; De Masi et al., 2019). This allows the study of the effects produced by the finite size population on the front propagation. We focus here on the third direction of research.

Brunet and Derrida (2001) highlighted that in contrast to the macroscopic equations, which admit infinitely many traveling waves (characterized by their velocities), microscopic models should select a unique velocity. Defining finite population particle systems, of size $N$, with branching and selection (in discrete time), they showed using heuristic arguments and simulations that the asymptotic speed of a particle system has a deviation of order $(\log N)^{-2}$ from the macroscopic speed. This sheds light on the effects of the finite size population, as the corresponding speed converges much slower than expected. This was rigorously proved in Bérard and Gouéré (2010) for $N$-Branching Random Walks ( $N$-BRW), a system in which particles branch, perform random walks and undergo a selection mechanism.

Several similar particle systems involving diffusive movements and sequential steps of branching and selection have been considered in this context. In Durrett and Remenik (2011) the authors consider a model closely related to $N-$ BRW and prove that the distribution function of the empirical measure converges, for fixed times, to a free boundary integro-differential equation in the F-KPP class, as well as proving a speed selection phenomenon. The same conclusions were conjectured to hold in Groisman and Jonckheere (2019) for the $N$-Branching Brownian motion $(N$-BBM $)$, introduced and studied in Maillard (2016). These conclusions have been recently settled in Berestycki et al. (2019); De Masi et al. (2019). More on this macroscopic equation can be found in Berestycki et al. (2018).

In the present article, we define and study a finite-population particle system in continuous time, closely following the dynamics proposed by Brunet and Derrida in Brunet and Derrida (2001). In our setting $N$ particles diffuse independently according to one dimensional Brownian motion, branch and get selected, with the important feature that particles are paired at each branching event. At these times, the particle in the pair with largest position branches into two and the one (in the pair) with smallest position is eliminated from the system. This corresponds to the branching-selection mechanism.

We first prove that the cumulative distribution function of the empirical measure of this system converges to a solution of the F-KPP equation as the number of particles goes to infinity. As a consequence, we are able to prove that the system selects the minimal speed of the macroscopic model as $N$ goes to infinity. In order to do that, we first show the existence of a stationary regime for the system seen from its leftmost particle and the existence of an asymptotic speed for fixed $N$, using classical arguments. Combining this with the hydrodynamic limit previously 
obtained, we get a lower bound for the speed. The upper bound can be obtained by constructing our process as a pruning of $N$ independent BBMs.

Some particular features of our setting, in contrast with previously mentioned works are (i) the proofs do not depend on explicit computations but rather on a careful analysis of the system (for instance, the limiting speed is obtained through comparison with F-KPP solutions and not through Legendre transforms), (ii) we provide explicit bounds for the decorrelation between particles and hence we can quantify the propagation of chaos, (iii) we believe that the analysis is robust enough to be extended to more general diffusions and branching-selection mechanisms (see Groisman and Soprano-Loto, 2020) and (iv) the behavior of the system as $N$ goes to infinity is given exactly by the solution of the F-KPP and not by approximations.

The rest of the paper is organized as follows. In Section 2, we introduce the model and state the main results. The proof of propagation of chaos and convergence towards the F-KPP is given in Section 3. Finally in Section 4, we prove the existence and properties of the velocities for fixed $N$ and then proceed to prove the selection principle: as $N$ goes to infinity, the velocities converge to the minimal velocity of the F-KPP equation.

\section{Model and main results}

We first describe the dynamics in an informal way. The system starts with $N$ particles located in the real line, each of which independently performs a standard Brownian motion and carries a Poisson clock with rate one. At Poissonian times, each particle chooses another particle uniformly among the other ones. Between these two particles, the one which has smaller position jumps on top of the other one. Throughout the article we will refer indistinctly to this interaction as a jump of the smaller particle of the pair to the position of the larger one or as a branching of the larger particle with killing of the smaller one. More precisely $\left(\xi_{t}\right)_{t \geqslant 0}$ is a Markov process in $\mathbb{R}^{N}$ with generator given by

$$
\mathcal{L} f(\xi)=\frac{1}{2} \sum_{i=1}^{N} \partial_{x_{i}}^{2} f(\xi)+\frac{1}{2(N-1)} \sum_{i=1}^{N} \sum_{j \neq i}\left(f\left(\theta_{i j}(\xi)\right)-f(\xi)\right),
$$

for $f \in C_{0}^{2}\left(\mathbb{R}^{N}\right)$, the set of twice differentiable functions with compact support. Here

$$
\theta_{i j}(\xi)(k)= \begin{cases}\max \{\xi(j), \xi(i)\} & \text { if } k \in\{i, j\} \\ \xi(k) & \text { otherwise. }\end{cases}
$$

Namely, given two labels $i, j, \theta_{i j}$ replaces the position of the particle with smaller position with the position of the larger one.

We denote by $\xi_{t}=\left(\xi_{t}(1), \ldots, \xi_{t}(N)\right)$ the process at time $t$ and define the empirical measure associated to $\xi_{t}$ by

$$
\mu_{t}^{N}:=\frac{1}{N} \sum_{i=1}^{N} \delta_{\xi_{t}(i)}, \quad t>0 .
$$

The cumulative distribution function of $\mu_{t}^{N}$ is given by,

$$
F_{N}(x, t):=\mu_{t}^{N}((-\infty, x]), \quad x \in \mathbb{R}, t>0 .
$$

For simplicity, we omit the dependence of $\mu_{t}^{N}$ and $F_{N}(x, t)$ on $\xi_{t}$ if not necessary. 
Notation. In what follows, we denote by $\mathcal{P}$ the space of probability measures on $\mathbb{R}$ endowed with the weak topology. For a polish metric space $S, D([0, T], S)$ is the space of càdlàg functions from $[0, T]$ to $S$ endowed with the Skorokhod topology. We assume that all our processes are defined in a probability space $(\Omega, \mathcal{F}, P)$ and denote with $E$, the expectation with respect to $P$ and with $V$ the variance with respect to $P$. When needed, we use $P_{\mu}$ (respectively $P_{\xi_{0}}$ ) whenever the initial condition is distributed at random according to the probability measure $\mu$ (respectively $\delta_{\xi_{0}}$ ), with the same convention for expectation and variance. The $\sigma$-algebra generated by the process up to time $t$ is denoted by $\mathcal{F}_{t}$. For simplicity, we write $u_{N}(x, t):=E_{\xi_{0}}\left[F_{N}(x, t)\right]$ and $\|\cdot\|=\|\cdot\|_{\infty}$ for the infinity norm of a real valued function.

2.1. Hydrodynamic limit. The following theorem provides a link between the aforementioned model and the solutions of the F-KPP equation, through the hydrodynamic limit.

Theorem 2.1. Let $u_{0}$ be a distribution function in $\mathbb{R}$ such that $\lim _{N \rightarrow \infty} \| F_{N}(\cdot, 0)$ $u_{0} \|=0$, a.s. and $u$ the solution to (1.1). Then, for any $t>0$ we have

$$
\lim _{N \rightarrow \infty}\left\|F_{N}(\cdot, t)-u(\cdot, t)\right\|=0 \quad \text { a.s. }
$$

We can also state a path-wise version of this result.

Corollary 2.2. In the setting of Theorem 2.1, let $\mu_{t}$ be the deterministic measure in $\mathbb{R}$ given by $\mu_{t}((-\infty, x])=u(x, t)$. Then,

$$
\lim _{N \rightarrow \infty}\left(\mu_{t}^{N}\right)_{t \geqslant 0}=\left(\mu_{t}\right)_{t \geqslant 0},
$$

weakly in $D([0,+\infty), \mathcal{P})$, in probability.

2.2. Speed selection. For $\xi \in \mathbb{R}^{N}$, let $\xi[i]$ denote the $i$-th order statistic of $\xi$, i.e.

$$
\begin{gathered}
\xi[1]=\min _{1 \leqslant j \leqslant N} \xi(j), \quad j_{1}=\underset{1 \leqslant j \leqslant N}{\arg \min } \xi(j), \\
\xi[i+1]=\min _{j \neq j_{k}, k \leqslant i} \xi(j) \quad j_{i+1}=\underset{j \neq j_{k}, k \leqslant i}{\arg \min } \xi(j) .
\end{gathered}
$$

We use the label of the particles to break ties. The position of the $i$-th particle as seen from the minimum is given by $\eta_{t}(i)=\xi_{t}[i+1]-\xi_{t}[1]$ and the process as seen from the minimum is defined by

$$
\eta_{t}=\left(\eta_{t}(1), \ldots, \eta_{t}(N-1)\right)
$$

Theorem 2.3. Let $N \geqslant 1$. Then,

(1) The process $\left(\eta_{t}\right)_{t \geqslant 0}$ has a unique stationary distribution $\nu^{N}$, which is absolutely continuous with respect to the $N$-dimensional Lebesgue measure.

(2) For any initial distribution $\rho_{0}^{N}$

$$
\lim _{t \rightarrow \infty}\left\|P_{\rho_{0}^{N}}\left(\eta_{t} \in \cdot\right)-\nu^{N}(\cdot)\right\|_{T V}=0
$$

(3) There exists $v_{N} \geqslant 0$ such that

$$
\lim _{t \rightarrow \infty} \frac{\xi_{t}[1]}{t}=\lim _{t \rightarrow \infty} \frac{\xi_{t}[N]}{t}=v_{N}, \quad \text { almost surely and in } L^{1} .
$$

(4) $v_{N+1} \geqslant v_{N}$ and

$$
\lim _{N \rightarrow \infty} v_{N}=\sqrt{2}
$$


Remark. Equation (2.6) can be thought as a selection principle in the sense that though the macroscopic equation admits infinitely many traveling waves with different velocities, for each $N$ and for any initial distribution the microscopic system has a unique velocity which converges to the minimal velocity of the macroscopic equation, when $N$ goes to infinity. This kind of result was first proved in Bramson et al. (1986).

Comment. Two open problems arise naturally from Theorem 2.3. The first one is whether a 'strong selection principle' holds, i.e whether under $\nu^{N}$ the cumulative distribution function of the empirical measure converges to the minimal traveling wave rather than just the velocities. We conjecture that this is in fact the case but we could not prove it. The main difficulty is that the domain of attraction of the minimal traveling wave is very thin. The solution of the F-KPP with initial data with heavier tails than the minimal traveling wave do not converge to the minimal traveling wave. Even if they have exponential decay at infinity (McKean, 1975). A similar statement has been proved in Asselah et al. (2016) in the context of quasistationary distributions and Fleming-Viot processes (a counterpart of these models, as observed in Groisman and Jonckheere, 2019). In that case, the minimal quasistationary distribution attracts every initial data with a finite mean and this is key to get the result. The second problem is related to the order of convergence of $v_{N}$ to $\sqrt{2}$. As conjectured by Brunet and Derrida in their models, we also expect here a slow rate of convergence of order $(\log N)^{-2}$. Naive simulations show such a slow rate (slower than any power of $N$ ), but our simulations are not precise enough to predict the exact order of convergence. Also, more involved computations to prove the lower bound for $\lim \inf v_{N}$ combined with the algebraic order of convergence conjectured for the convergence to the minimal traveling wave in the F-KPP might allow to get a (power of a) logarithmic order of convergence. This would just give lower bound estimates though, which is not very informative.

\section{Hydrodynamic limit}

We begin now with the proof of Theorem 2.1. The main idea is to show that $u_{N}$ verifies, in the limit as $N$ goes to infinity, the F-KPP equation and that the variances $V_{\xi_{0}}\left[F_{N}(x, t)\right]$ converge to zero, which boils down to proving asymptotic decorrelation of the particles (propagation of chaos). The following graphical construction will turn to be instrumental for this purpose.

3.1. Graphical construction. We construct the process as a deterministic function of $N$ independent Brownian motions and an homogeneous Poisson process (for the jumps).

To each particle $i$, we associate a marked Poisson process $\omega^{i}$ and an independent standard Brownian motion $\left(B_{t}^{i}\right)_{t \geqslant 0}$. The process $\omega^{i}$ is defined on $\mathbb{R} \times$ $(\{1, \ldots, N\} \backslash\{i\})$ with intensity measure $d t d \beta_{i}$, where $\beta_{i}$ is the uniform distribution on $\{1, \ldots, N\} \backslash\{i\}$. The processes $\left(\omega^{i},\left(B_{t}^{i}\right)_{t \geqslant 0}\right)_{1 \leqslant i \leqslant N}$ are independent. Consider the superposition $\omega=\bigcup \omega^{i} \times\{i\}$ and sort the marks in order of appearance. We denote this sequence with $\left(\tau_{k}, j_{k}, i_{k}\right)_{k \in \mathbb{N}}$ where the first coordinate $\tau$ is the time mark, the second one is the partner mark chosen according to $\beta_{i}$ and the third one is the label mark (the label mark is $i$ if it occurs at $\omega^{i}$ ). We define $\xi_{t}$ inductively as follows.

- At time 0 , the configuration is $\xi_{0}$. 
- Assume $\xi_{\tau_{k}}$ is defined. For $t \in\left(\tau_{k}, \tau_{k+1}\right]$ we define

$$
\xi_{t}(l)= \begin{cases}\xi_{t^{-}}\left(j_{k+1}\right) & \text { if } t=\tau_{k+1}, l=i_{k+1} \text { and } \xi_{t^{-}}\left(i_{k+1}\right)<\xi_{t^{-}}\left(j_{k+1}\right) \\ \xi_{\tau_{k}}(l)+B_{t}^{l}-B_{\tau_{k}}^{l} & \text { otherwise. }\end{cases}
$$

It is straightforward to check that $\left(\xi_{t}\right)_{t \geqslant 0}$, as constructed above is Markov, with generator given by (2.1).

For each particle $i$ and a time $t>0$, we construct backwards a set of (labels of) particles that we call ancestors. The important feature of this set is that the position of the particle is measurable with respect to the $\sigma$-algebra generated by the Brownian motions and Poisson processes attached to the ancestors up to time $t$. For any two particles, conditioned on the event that their clans of ancestors do not intersect, the particles are independent. More details below.

3.2. The clan of ancestors. Given a set of labels $A \subseteq\{1, \ldots, N\}$ we denote by $\omega^{A}[0, s]$ the superposition of all the points of $\omega^{j}$ with $j \in A$ restricted to the interval $[0, s]$. We add $(0,0,0)$ to this set for convenience. That is, $\omega^{A}[0, s]=$ $\bigcup_{j \in A} \omega^{j}([0, s]) \bigcup\{(0,0,0)\}$. For each $1 \leqslant i \leqslant N$, we define inductively a sequence of times and sets as follows.

- Let $s_{1}$ be the largest time mark in $\omega^{\{i\}}[0, t]$ and $j_{1}$ its partner mark. If $s_{1}=0$ stop and define $\psi_{t}^{i}=\{i\}$. Otherwise define $A_{1}^{i}=\left\{i, j_{1}\right\}$.

- Suppose $s_{k}, A_{k}^{i}$ are defined. Then, let $s_{k+1}$ be the largest time in $\omega^{A_{k}^{i}}\left[0, s_{k}\right)$ and $j_{k+1}$ its partner mark. If $s_{k+1}=0$ stop and define $\psi_{t}^{i}=A_{k}^{i}$. Otherwise define $A_{k+1}^{i}=A_{k}^{i} \cup\left\{j_{k+1}\right\}$.

As $N$ is finite, this procedure finishes after a finite number of steps $n_{t}^{i}$ almost surely.

The next two lemmas give a bound on the probability of intersection of two clans of ancestors and, as a consequence, a bound for the two-particles correlation. Their proofs are essentially contained in Asselah et al. (2011, Lemma 2.1 and Section 3). We include a sketch of them for the reader's convenience.

Lemma 3.1. For $i \neq j$ and $t>0$,

$$
P_{\mu_{0}^{N}}\left(\psi_{t}^{i} \cap \psi_{t}^{j} \neq \varnothing\right) \leqslant \frac{e^{2 t}-1}{N-1} .
$$

Sketch of the proof. Observe that the process $\mathbf{1}\left\{\psi_{t}^{i} \cap \psi_{t}^{j} \neq \varnothing\right\}$ jumps from 0 to 1 at rate $\frac{\left|\psi_{t}^{i}\right|\left|\psi_{t}^{j}\right|}{2(N-1)}$. We thus get,

$$
\partial_{t} P\left(\psi_{t}^{i} \cap \psi_{t}^{j} \neq \varnothing\right)=E\left[\mathbf{1}\left\{\psi_{t}^{i} \cap \psi_{t}^{j} \neq \varnothing\right\} \frac{\left|\psi_{t}^{i}\right|\left|\psi_{t}^{j}\right|}{(N-1)}\right] \leqslant \frac{E\left[\left|\psi_{t}^{i}\right|\right] E\left[\left|\psi_{t}^{j}\right|\right]}{N-1} .
$$

The growth rate of $\psi_{t}^{i}$ at time $t$ is precisely $\left|\psi_{t}^{i}\right|$, which implies that $E\left(\left|\psi_{t}^{i}\right|\right)=e^{t}$ and the lemma follows.

In the event $\left\{\psi_{t}^{i} \cap \psi_{t}^{j}=\varnothing\right\}$ the only possible dependence between $\xi_{t}^{i}$ and $\xi_{t}^{j}$ is due to the initial condition. As a consequence, for deterministic initial conditions, we obtain the following bound. 
Lemma 3.2. For $t \geqslant 0$ and $x, y \in \mathbb{R}$, we have

$$
\begin{aligned}
& \sup _{\xi \in \mathbb{R}^{N}}\left|\sum_{i, j=1}^{N} E_{\xi}\left[\mathbf{1}\left\{\xi_{t}(i) \leqslant x\right\} \mathbf{1}\left\{\xi_{t}(j) \leqslant y\right\}\right]-E_{\xi}\left[\mathbf{1}\left\{\xi_{t}(i) \leqslant x\right\}\right] E_{\xi}\left[\mathbf{1}\left\{\xi_{t}(j) \leqslant y\right\}\right]\right| \\
& \leqslant 2 N e^{2 t} .
\end{aligned}
$$

Proof: Let us observe that

$$
\begin{aligned}
& P_{\xi}\left(\xi_{t}(i) \leqslant x, \xi_{t}(j) \leqslant y\right)= \\
& P_{\xi}\left(\xi_{t}(i) \leqslant x, \xi_{t}(j) \leqslant y ; \psi_{t}^{i} \cap \psi_{t}^{j} \neq \varnothing\right)+P_{\xi}\left(\xi_{t}(i) \leqslant x, \xi_{t}(j) \leqslant y ; \psi_{t}^{i} \cap \psi_{t}^{j}=\varnothing\right)= \\
& P_{\xi}\left(\xi_{t}(i) \leqslant x, \xi_{t}(j) \leqslant y ; \psi_{t}^{i} \cap \psi_{t}^{j} \neq \varnothing\right)+\sum_{a \cap b=\varnothing} P_{\xi}\left(\xi_{t}(i) \leqslant x, \xi_{t}(j) \leqslant y ; \psi_{t}^{i}=a, \psi_{t}^{j}=b\right)= \\
& P_{\xi}\left(\xi_{t}(i) \leqslant x, \xi_{t}(j) \leqslant y ; \psi_{t}^{i} \cap \psi_{t}^{j} \neq \varnothing\right)+\sum_{a \cap b=\varnothing} P_{\xi}\left(\xi_{t}(i) \leqslant x, \psi_{t}^{i}=a\right) P_{\xi}\left(\xi_{t}(j) \leqslant y, \psi_{t}^{j}=b\right),
\end{aligned}
$$

while

$$
\begin{aligned}
& P_{\xi}\left(\xi_{t}(i) \leqslant x\right) P_{\xi}\left(\xi_{t}(j) \leqslant y\right)=P_{\xi}\left(\xi_{t}(i) \leqslant x, \tilde{\xi}_{t}(j) \leqslant y\right)= \\
& P_{\xi}\left(\xi_{t}(i) \leqslant x, \tilde{\xi}_{t}(j) \leqslant y ; \psi_{t}^{i} \cap \tilde{\psi}_{t}^{j} \neq \varnothing\right)+P_{\xi}\left(\xi_{t}(i) \leqslant x, \tilde{\xi}_{t}(j) \leqslant y ; \psi_{t}^{i} \cap \tilde{\psi}_{t}^{j}=\varnothing\right)= \\
& P_{\xi}\left(\xi_{t}(i) \leqslant x, \tilde{\xi}_{t}(j) \leqslant y ; \psi_{t}^{i} \cap \tilde{\psi}_{t}^{j} \neq \varnothing\right)+\sum_{a \cap b=\varnothing} P_{\xi}\left(\xi_{t}(i) \leqslant x, \tilde{\xi}_{t}(j) \leqslant y ; \psi_{t}^{i}=a, \tilde{\psi}_{t}^{j}=b\right)= \\
& P_{\xi}\left(\xi_{t}(i) \leqslant x, \tilde{\xi}_{t}(j) \leqslant y ; \psi_{t}^{i} \cap \tilde{\psi}_{t}^{j} \neq \varnothing\right)+\sum_{a \cap b=\varnothing} P_{\xi}\left(\xi_{t}(i) \leqslant x, \psi_{t}^{i}=a\right) P_{\xi}\left(\tilde{\xi}_{t}(j) \leqslant y, \tilde{\psi}_{t}^{j}=b\right),
\end{aligned}
$$

with $\tilde{\xi}_{t}$ an independent copy of $\xi_{t}$. Substracting (3.1) and (3.2) we get that for $i \neq j$,

$$
\begin{aligned}
\mid P_{\xi}\left(\xi_{t}(i) \leqslant x, \xi_{t}(j) \leqslant y\right) & -P_{\xi}\left(\xi_{t}(i) \leqslant x\right) P_{\xi}\left(\xi_{t}(j) \leqslant y\right) \mid \\
& \leqslant P\left(\psi_{t}^{i} \cap \psi_{t}^{j} \neq \varnothing\right)+P\left(\psi_{t}^{i} \cap \tilde{\psi}_{t}^{j} \neq \varnothing\right) \leqslant 2 \frac{e^{2 t}-1}{N-1},
\end{aligned}
$$

where in the last inequality we make use of Lemma 3.1. Thus, by summing over $i$ and $j \in\{1, \ldots, N\}$, and noting that there are $N$ diagonal terms which bring a factor $N$ when $i=j$, we get the desired bound.

The following lemma allows us to control perturbations of the F-KPP equation.

Lemma 3.3. Let $u$ be a solution of (1.1) with initial datum $u_{0}$ and $w$ a smooth bounded function in $\mathbb{R} \times[0, T]$ such that

$$
\partial_{t} w=\frac{1}{2} \partial_{x}^{2} w+w^{2}-w+g, \quad 0 \leqslant w(x, 0)=w_{0}(x) \leqslant 1 .
$$

Then

$$
\|u(\cdot, t)-w(\cdot, t)\| \leqslant\left(\left\|u_{0}-w_{0}\right\|+\int_{0}^{t} e^{-s}\|g(\cdot, s)\| d s\right) e^{2 t},
$$

for $\|g\|$ small enough (depending on $T$ ).

Proof: By the maximum principle for parabolic equations in unbounded domains (Quittner and Souplet, 2007, Proposition 52.4) we get $\|w\| \leqslant 2$ for $\|g\|$ small enough. This can be obtained through the ODE

$$
\dot{y}=y^{2}-y+\|g\|, \quad y(0)=1 .
$$


Since $y$ is a bound from above for $\|w\|$ we just need to bound $y$. Observe that $y^{2}-y \leqslant 2(y-1)$ for $0 \leqslant y \leqslant 2$, i.e. for $t \leqslant t^{*}:=\inf \{s \geqslant 0: y(s) \leqslant 2\}$. We will prove that $t^{*}>T$ for $\|g\|$ small enough. Solving the linear equation $\dot{\bar{y}}=2(\bar{y}-1)+\|g\|$ we get that for $t<t^{*}, y(t) \leqslant 1-\|g\| / 2+\frac{\|g\|}{2} e^{2 t}$. This implies that $t^{*} \rightarrow \infty$ as $\|g\| \rightarrow 0$ and the bound follows. Call $\underline{z}=w-u$ and

$$
\bar{z}(x, t)=\left(\left\|u_{0}-w_{0}\right\|+\int_{0}^{t} e^{-s}\|g(\cdot, s)\| d s\right) e^{2 t} .
$$

Hence, this function verifies

$$
\begin{aligned}
\partial_{t} \bar{z} & =\frac{1}{2} \partial_{x}^{2} \bar{z}+2 \bar{z}+e^{t}\|g(\cdot, t)\| \\
& \geqslant \frac{1}{2} \partial_{x}^{2} \bar{z}+(w+u-1) \bar{z}+\|g(\cdot, t)\|,
\end{aligned}
$$

with $\bar{z}(x, 0)=\left\|u_{0}-w_{0}\right\|$, while for $\underline{z}$ we have

$$
\begin{aligned}
\partial_{t} \underline{z} & =\frac{1}{2} \partial_{x}^{2} \underline{z}+w^{2}-u^{2}-\underline{z}+g \\
& =\frac{1}{2} \partial_{x}^{2} \underline{z}+(w+u-1) \underline{z}+g .
\end{aligned}
$$

Hence $e=\underline{z}-\bar{z}$ verifies

$$
\partial_{t} e \leqslant \frac{1}{2} \partial_{x}^{2} e+(w+u-1) e
$$

With $e(x, 0)=w_{0}(x)-u_{0}(x)-\left\|u_{0}-w_{0}\right\| \leqslant 0$. Since $u$ and $w$ are bounded, using the maximum principle again we obtain $e(x, t) \leqslant 0$ for all $(x, t) \in \mathbb{R} \times[0, T]$. Proceeding in the same way with $-\bar{z}-\underline{z}$ we get that for all $(x, t) \in \mathbb{R} \times[0, T]$, $-\bar{z}(x, t) \leqslant w(x, t)-u(x, t) \leqslant \bar{z}(x, t)$, which conludes the proof.

We are ready to prove Theorem 2.1.

Proof of Theorem 2.1: Throughout this proof, we denote $E_{\xi_{0}}, V_{\xi_{0}}$ and $P_{\xi_{0}}$ briefly by $E, V$ and $P$, respectively. We also use $\mathcal{G}(x, t ; z)=\int_{-\infty}^{x}(2 \pi t)^{-1 / 2} e^{-(y-z)^{2} / 2 t} d y$ for the probability that a Brownian motion started at $z$ is less than $x$ at time $t$. Let us recall that

$$
u_{N}(x, t):=E_{\xi_{0}}\left[F_{N}(x, t)\right] .
$$

The proof is divided into two steps.

Step 1. Derivation of the equation for $u_{N}(x, t)$.

Let us first observe that

$$
u_{N}(x, t)=\frac{1}{N} \sum_{i=1}^{N} P\left(\xi_{t}(i) \leqslant x\right) .
$$

We will focus on each term of the previous sum separately. Fix $i$ and let $\tau$ be the last time-mark before time $t$ of $\omega^{i}$. If there is no such a mark, we set $\tau=0$. Notice that particle $i$ performs a Brownian motion in $(\tau, t)$ and that $\tau \sim t-\min (Z, t)$ with $Z$ an exponential random variable with mean 1 . Thus, by conditioning on $\tau$ we get

$$
P\left(\xi_{t}(i) \leqslant x\right)=e^{-t} \mathcal{G}\left(x, t ; \xi_{0}(i)\right)+\int_{0}^{t} e^{-(t-s)} E\left[\mathcal{G}\left(x, t-s ; \xi_{s}(i)\right) \mid \tau=s\right] d s .
$$


Observe that for any $z, \mathcal{G}(\cdot, \cdot ; z)$ verifies the heat equation. We now differentiate the r.h.s. of the previous equality with respect to $t$ to obtain,

$$
\begin{aligned}
& -e^{-t} \mathcal{G}\left(x, t ; \xi_{0}(i)\right)+\frac{e^{-t}}{2} \partial_{x}^{2} \mathcal{G}\left(x, t ; \xi_{0}(i)\right)+\int_{0}^{t}-e^{-(t-s)} E\left[\mathcal{G}\left(x, t-s ; \xi_{s}(i)\right) \mid \tau=s\right] d s \\
& +\int_{0}^{t} e^{-(t-s)} E\left[\frac{1}{2} \partial_{x}^{2} \mathcal{G}\left(x, t-s ; \xi_{s}(i)\right) \mid \tau=s\right] d s+E\left[\mathcal{G}\left(x, 0 ; \xi_{t}(i)\right) \mid \tau=t\right] .
\end{aligned}
$$

Combining the first term with the third one and the second term with fourth one, we obtain that for every $1 \leqslant i \leqslant N$, the function $q_{i}(x, t):=P\left(\xi_{t}(i) \leqslant x\right)$ verifies,

$$
\partial_{t} q_{i}=\frac{1}{2} \partial_{x}^{2} q_{i}-q_{i}+P\left(\xi_{t}(i) \leqslant x \mid \tau=t\right)
$$

We proceed to examine the last term in (3.4). Let $J_{i j}$ be the event that the partner mark is $j$. Conditioning on $J_{i j}$ we get

$$
\begin{aligned}
P\left(\xi_{t}(i) \leqslant x \mid \tau=t\right) & =\sum_{j \neq i} P\left(\max \left(\xi_{t^{-}}(i), \xi_{t^{-}}(j)\right) \leqslant x \mid \tau=t, J_{i j}\right) P\left(J_{i j} \mid \tau=t\right) \\
& =\frac{1}{N-1} E\left[\sum_{j \neq i} \mathbf{1}\left\{\xi_{t}(i) \leqslant x\right\} \mathbf{1}\left\{\xi_{t}(j) \leqslant x\right\}\right] \\
& =\frac{1}{N-1} E\left[\sum_{j=1}^{N} \mathbf{1}\left\{\xi_{t}(i) \leqslant x\right\} \mathbf{1}\left\{\xi_{t}(j) \leqslant x\right\}-\mathbf{1}\left\{\xi_{t}(i) \leqslant x\right\}\right] .
\end{aligned}
$$

Since $F_{N}^{2}(x, t)=\frac{1}{N^{2}} \sum_{i, j=1}^{N} \mathbf{1}\left\{\xi_{t}(i) \leqslant x\right\} \mathbf{1}\left\{\xi_{t}(j) \leqslant x\right\}$, combining (3.3),(3.4) and (3.5) we get

$$
\begin{aligned}
\partial_{t} u_{N} & =\frac{1}{2} \partial_{x}^{2} u_{N}-\frac{N}{N-1} E\left[F_{N}\left(1-F_{N}\right)\right] \\
& =\frac{1}{2} \partial_{x}^{2} u_{N}-u_{N}\left(1-u_{N}\right)+\frac{N}{N-1} V\left(F_{N}(x, t)\right)-\frac{1}{N-1}\left(u_{N}\left(1-u_{N}\right)\right) .
\end{aligned}
$$

Next we prove that solutions of (3.6) converge to solutions of (1.1) when $N$ tends to infinity.

Step 2. Control of the variance and stability of the F-KPP.

We first observe that,

$$
\begin{aligned}
\left|F_{N}(x, t)-u(x, t)\right| & =\left|\frac{1}{N} \sum_{i=1}^{N} Z_{i}+u_{N}(x, t)-u(x, t)\right| \\
& \leqslant\left|\frac{1}{N} \sum_{i=1}^{N} Z_{i}\right|+\left|u_{N}(x, t)-u(x, t)\right|,
\end{aligned}
$$

with $Z_{i}:=\mathbf{1}\left\{\xi_{t}(i) \leqslant x\right\}-q_{i}(x, t)$. The Strong Law of Large numbers for weakly correlated variables (Lyons, 1988, Theorem 1) gives us $\lim _{N \rightarrow \infty} \frac{1}{N} \sum_{i=1}^{N} Z_{i}=0$ a.s., provided that the following two conditions are fulfilled:

(i) $E\left[\left|Z_{i}\right|^{2}\right] \leqslant 1$, and $\left|Z_{i}\right| \leqslant 1$ a.s. for all $i \in \mathbb{N}$, 
(ii) $\sum_{N \geqslant 1} \frac{1}{N} E\left[\left|\frac{1}{N} \sum_{i=1}^{N} Z_{i}\right|^{2}\right]<\infty$.

Condition (i) follows immediately. To check (ii), observe that by Lemma 3.2, we get that

$$
\sup _{\xi_{0}} E_{\xi_{0}}\left[\left|\frac{1}{N} \sum_{i=1}^{N} Z_{i}\right|^{2}\right]=\sup _{\xi_{0}} V_{\xi_{0}}\left(F_{N}(x, t)\right) \leqslant \frac{2}{N} e^{2 T} .
$$

For the second term in (3.7) we apply Lemma 3.3 with $w=u_{N}$ and $g=$ $\frac{N}{N-1} V\left(F_{N}(x, t)\right)-\frac{1}{N-1}\left(u_{N}\left(1-u_{N}\right)\right)$. Since $\|g\| \rightarrow 0$ as $N \rightarrow \infty$, for $N$ large enough we have

$$
\begin{aligned}
\left\|u_{N}(\cdot, t)-u(\cdot, t)\right\| & \leqslant\left(\left\|F_{N}(\cdot, 0)-u_{0}(\cdot)\right\|+\int_{0}^{t} e^{-s}\|g(\cdot, s)\| d s\right) e^{2 t} \\
& \leqslant\left(\left\|F_{N}(\cdot, 0)-u_{0}(\cdot)\right\|+\frac{1}{N-1}\left(1+2 e^{2 T}\right)\right) e^{2 T}
\end{aligned}
$$

for all $t \leqslant T$. In the last inequality we use that $0 \leqslant u_{N} \leqslant 1$ and (3.8). Since the first term goes to zero a.s., we conclude that

$$
\lim _{N \rightarrow \infty}\left\|u_{N}(\cdot, t)-u(\cdot, t)\right\|=0, \text { a.s. }
$$

Hence, for every $x \in \mathbb{R}$ and $t>0, F_{N}(x, t) \rightarrow u(x, t)$, a.s. Let $k>0$ fixed. Since $u(\cdot, t)$ is continuous and increasing, there is a finite family $\left(c_{i}\right)_{1 \leqslant i \leqslant k-1} \subset \mathbb{R}$ such that $u\left(c_{i}, t\right)=i / k$. Since $F_{N}(\cdot, t)$ is increasing:

$$
\left|F_{N}(x, t)-u(x, t)\right| \leqslant 1 / k+\max _{1 \leqslant i \leqslant k}\left|F_{N}\left(c_{i}, t\right)-u\left(c_{i}, t\right)\right|
$$

Using the a.s. convergence for points $\left(c_{i}\right)_{1 \leqslant i \leqslant k-1}$, we get uniform convergence of $F_{N}(\cdot, t)$ towards $u(\cdot, t)$.

Proof of Corollary 2.2. The main ingredient to obtain Corollary 2.2 is to show that for every $T>0$, the sequence $\left(\mu_{t}^{N}\right)_{0 \leqslant t \leqslant T}$ is tight in $D([0, T], \mathcal{P})$. Afterwards, Theorem 2.1 implies that there is a unique limit point and hence the desired result.

Tightness. To prove tightness of $\left(\mu_{t}^{N}\right)_{0 \leqslant t \leqslant T}$ it is enough to show that for any $\varphi \in C_{0}^{1}(\mathbb{R})$ (the space of functions with continuous derivative supported on a compact set) the sequence of real-valued processes $\left(\left\langle\mu_{t}^{N}, \varphi\right\rangle\right)_{0 \leqslant t \leqslant T}$, with $\left\langle\mu_{t}^{N}, \varphi\right\rangle=$ $\int_{-\infty}^{+\infty} \varphi(x) \mu_{t}^{N}(d x)$, is tight in $D([0, T], \mathbb{R})$, see Roelly-Coppoletta (1986, Theorem $2.1)$.

According to Aldous criterion (Kallenberg, 2002, Theorem 16.11 and Lemma 16.12) the following two conditions are enough to get tightness for the previous sequence:

(i) For every $t \in[0, T] \cap \mathbb{Q}$ and every $\varepsilon>0$, there is an $L>0$ such that

$$
\sup _{N>0} P\left(\left|\left\langle\mu_{t}^{N}, \varphi\right\rangle\right|>L\right) \leqslant \varepsilon \text {. }
$$

(ii) Let $\mathfrak{T}_{T}^{N}$ be the collection of stopping times with respect to the natural filtration associated to $\left\langle\mu_{t}^{N}, \varphi\right\rangle$ that are almost surely bounded by $T$. For 
every $\varepsilon>0$

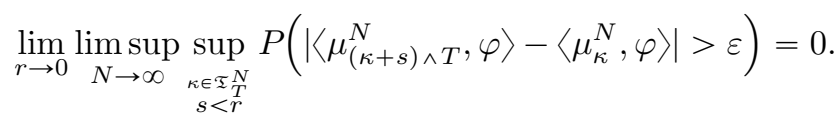

The first condition follows immediately by taking $L>\|\varphi\|$. For the second one, let $A_{i}:=\left\{\omega^{i}[\kappa, \kappa+s] \neq \varnothing\right\}$ and observe that for every $1 \leqslant i \leqslant N$,

$$
\begin{aligned}
E\left[\left|\varphi\left(\xi_{\kappa+s}^{i}\right)-\varphi\left(\xi_{\kappa}^{i}\right)\right|\right] & \leqslant 2\|\varphi\| P\left(A_{i}\right)+E\left[\left|\varphi\left(\xi_{\kappa}^{i}+B_{\kappa+s}^{i}-B_{\kappa}^{i}\right)-\varphi\left(\xi_{\kappa}^{i}\right)\right| \mathbf{1}\left\{A_{i}^{c}\right\}\right] \\
& \leqslant 2\|\varphi\|\left(1-e^{-s}\right)+E\left[\left|\varphi\left(\xi_{\kappa}^{i}+\tilde{B}_{s}\right)-\varphi\left(\xi_{\kappa}^{i}\right)\right|\right] \\
& \leqslant 2\|\varphi\|\left(1-e^{-s}\right)+\left\|\varphi^{\prime}\right\| E\left[\left|\tilde{B}_{s}\right|\right]
\end{aligned}
$$

as $s \rightarrow 0$ by dominated convergence. Here $\tilde{B}_{s}$ is an independent Brownian motion. By means of Markov inequality

$$
P\left(\left|\left\langle\mu_{(\kappa+s) \wedge T}^{N}, \varphi\right\rangle-\left\langle\mu_{\kappa}^{N}, \varphi\right\rangle\right|>\varepsilon\right) \leqslant \frac{\sum_{i=1}^{N} E\left[\left|\varphi\left(\xi_{\kappa+s}^{i}\right)-\varphi\left(\xi_{\kappa}^{i}\right)\right|\right]}{N \varepsilon} \leqslant \frac{2 r\left(\|\varphi\|+\left\|\varphi^{\prime}\right\|\right)}{\varepsilon} .
$$

This concludes the proof of (ii).

Uniqueness of limit points. Let $\left(\mu_{t}^{N_{k}}\right)_{0 \leqslant t \leqslant T}$ be any convergent subsequence and $\left(\mu_{t}\right)_{0 \leqslant t \leqslant T}$ its limit. Its time marginals $\mu_{t}$ are characterized by their distribution functions $F_{\mu_{t}}$. Due to Theorem 2.1 we have that $F_{\mu_{t}}=u(\cdot, t)$. This, together with Billingsley (1999, Theorem 13.1) provide us the desired conclusion.

\section{Speed selection}

In this section, we prove Theorem 2.3. The proofs for fixed $N$ are similar to the ones in Durrett and Remenik (2011); Bérard and Gouéré (2010). We include them for the reader's convenience but we point to those references for the details. However, the proof of convergence of the velocities $v_{N} \nearrow \sqrt{2}$ as $N \rightarrow \infty$ requires a completely different strategy and is based on the hydrodynamic limit, Theorem 2.1.

Parts (1) and (2) of Theorem 2.3 are obtained by proving positive Harris recurrence for the process $\left(\eta_{t}\right)_{t \geqslant 0}$ and part (3) by means of the subadditive ergodic theorem. The monotonicity of the velocities (4), is proved by coupling two process with different number of particles but comparable initial conditions. With some abuse of notation we will denote $\left(\eta_{k}\right)_{k \geqslant 0}$ a process constructed in this section which coincides in law with $\left(\eta_{t}\right)_{t \geqslant 0}$ observed at jump times, although the construction is different.

In what follows we omit the dependence of some of the variables on $N$. Consider a Poisson process $\left(T_{k}\right)_{k \geqslant 1}$ with rate $\frac{N}{2}$ and an i.i.d. family $\left(V_{k}\right)_{k \geqslant 1}$ with $V_{k}=$ $\left(V_{k}^{1}, V_{k}^{2}\right) \sim \mathcal{U}\left\{O_{N}\right\}$, where $O_{N}=\{(i, j): 1 \leqslant i<j \leqslant N\}$. Let $\left(B_{s}^{N}\right)_{s \geqslant 0}$ be an $N$-dimensional standard Brownian motion. For $\xi \in \mathbb{R}^{N}$ we denote $\sigma(\xi)=$ $(\xi[1], \ldots, \xi[N])$.

We construct a discrete time auxiliary Markov process $\zeta_{k}$ as follows. Given $\zeta_{0}$, define

$$
\zeta_{k+1}:=\sigma\left(\theta_{V_{k}}\left(\sigma\left(\zeta_{k}+B_{T_{k+1}}^{N}-B_{T_{k}}^{N}\right)\right)\right)
$$

with $\theta_{i j}$ defined in (2.2) and assuming $T_{0}=0$. In words, between jump times, particles evolve according to an $N$-dimensional Brownian motion. At jump times, two ranks $V=\left(V^{1}, V^{2}\right)$ are chosen uniformly in $O_{N}$ and the particle with rank $V^{1}$ 
jumps to the position of the particle with rank $V^{2}$. Afterwards, particles are sorted in increasing order. We omit the trajectories between jumps, so that $\zeta_{k}$ is the state of the system at the $k$-th jump of $\left(\xi_{t}\right)$.

The law of $\left(\zeta_{k}\right)_{k \geqslant 0}$ is given by the order statistics of a process with generator (2.1) at jump times as can be seen by direct computation. This process is a deterministic function of the initial condition $\zeta_{0}$, the displacements $D_{k}:=B_{T_{k}}^{N}-B_{T_{k-1}}^{N}$ and the jump marks $\left(V_{k}\right)_{k \geqslant 1}$. We emphasize this by writing

$$
\left(\zeta_{k}\right)_{k \geqslant 0}=\Upsilon\left(\left(V_{k}\right)_{k \geqslant 1},\left(D_{k}\right)_{k \geqslant 1}, \zeta_{0}\right) .
$$

As in (2.4), let $\left(\eta_{k}\right)_{k \geqslant 0}$ be the process $\left(\zeta_{k}\right)_{k \geqslant 0}$ as seen from the leftmost particle and $\Omega_{N}:=\left\{\left(z_{1}, \ldots, z_{N-1}\right) \in \mathbb{R}^{N-1}: 0 \leqslant z_{1} \leqslant \ldots \leqslant z_{N-1}\right\}$ its state space. Any set $R \subseteq \Omega_{N}$ is embedded in $\mathbb{R}^{N}$ by defining $R^{0}=\{0\} \times R$. We now turn to prove each of the statements stated in Theorem 2.3.

4.1. Positive Harris recurrence. To prove Positive Harris recurrence it is sufficient (see for instance Asmussen, 2003) to show that there exist a set $R \subseteq \Omega_{N}$ such that

(i) $E_{\eta}\left(\tau_{R}\right)<\infty$, for all $\eta \in \Omega_{N}$, where $\tau_{R}=\inf \left\{k \geqslant 0: \eta_{k} \in R\right\}$.

(ii) There exists a probability measure $q$ on $R, \lambda>0$ and $r \in \mathbb{N}$ so that $P_{\eta_{0}}\left(\eta_{r} \in \tilde{R}\right) \geqslant \lambda q(\tilde{R})$ for all $\eta_{0} \in R$ and all $\tilde{R} \subseteq R$.

Take

$$
R=\left\{\eta \in \Omega_{N}: \eta(i+1)-\eta(i) \in(0, N) \text { for } i=1, \ldots, N-1\right\} .
$$

We claim that any initial condition $\eta \in \Omega_{N}$ can be driven by the dynamics into the set $R$ in exactly $N-1$ steps with a positive probability uniformly bounded below away from zero. In fact that is the case if (a) each particle does not displace more than $1 / 2$ between jumps (due to Brownian movement) and (b) the particles chosen by $\left(V_{k}\right)_{k \geqslant 1}$ to perform the jumps are always the first and the last one (hence the first particle jumps to the position of the last one). In this event we have that $\eta_{N-1} \in R$.

The probability of this event can be bounded below uniformly on the initial condition by

$$
P_{\eta}\left(\eta_{N-1} \in R\right) \geqslant\left(a^{N} P\left(V_{1}=(1, N)\right)\right)^{N-1}=\left(\frac{2 a^{N}}{N(N-1)}\right)^{N-1},
$$

with $a>0$ being the probability that a Brownian motion started at the origin does not leave $(-1 / 2,1 / 2)$ before a time given by an independent exponential random variable with parameter $N-1$. This implies (i).

To prove (ii) observe that the displacement of the particles between jumps has the law of the order statistics for $N$ independent Brownian motions, whose density is uniformly bounded below for any initial condition $\eta_{0} \in R$ by a positive constant $c_{N}$. Taking $q$ as the normalized Lebesgue measure restricted to $R, r=1$ and $\lambda=c_{N} e^{-N}$ (the factor $e^{-N}$ stands for the probability of not having jumps in $[0,1]$ ) we get that (ii) holds, which completes the proof of (ii).

It is easy to check, by means of (4.3) and the strong Markov property, that $\sup _{\eta} E_{\eta}\left(\tau_{R}\right)<\infty$ and hence $\left(\eta_{k}\right)_{k \geqslant 0}$ is positive Harris recurrent, which implies that a unique invariant measure exists and is finite. The fact that $\nu^{N}$ is absolutely continuous follows from the fact that the distribution of the process is absolutely continuous for every positive time, for every initial distribution. 
4.2. Convergence to equilibrium. To prove (2) it suffices to show the result for the subsequences $\left(\eta_{N m+j}\right)_{m \geqslant 0}$, with $0 \leqslant j<N$. Furthermore, due to the Markov property, we only need to consider the case $j=0$. The inequality obtained in (4.3) (which is independent of $\eta$ ) implies that $\sup _{\eta} P_{\eta}\left(\tau_{R}>t\right)<1$ for some $t>0$. The result follows by applying Theorem 4.1(ii) in Athreya and Ney (1978) to the subsequence $\left(\eta_{N m}\right)_{m \geqslant 0}$.

4.3. Existence of a velocity. We prove that both limits in (2.5) exist almost surely and in $L^{1}$ and that they are nonrandom as in Bérard and Gouéré (2010). The argument makes use of the subadditive ergodic theorem Durrett (1991). First observe that due to the monotonicity property (with respect to the initial state) of the coupling introduced in (4.1) and translation invariance, it is sufficient to prove the result when all the particles start at the origin.

We construct several copies of our process simultaneously. The index $n$ refers to the $n$-th copy and the index $k$ refers to time. Given the sequences $\left(D_{k}\right)_{k \geqslant 1}$ for the displacements and $\left(V_{k}\right)_{k \geqslant 1}$ for the jumps we construct each copy of the process in the following manner:

$$
\zeta_{n, 0}=\xi_{0}, \quad\left(\zeta_{n, k}\right)_{k \geqslant 1}=\Upsilon\left(\left(V_{r}\right)_{r \geqslant n},\left(D_{r}\right)_{r \geqslant n}, \zeta_{n, 0}\right), \quad n \geqslant 0 .
$$

Here $\Upsilon$ refers to the construction introduced in (4.2). Let us stress the fact that the $n$-th copy of the process does not make use of the first $n$ elements of the sequences $\left(V_{r}\right)_{r \geqslant 1},\left(D_{r}\right)_{r \geqslant 1}$. Thus, for $d \in \mathbb{N},\left(\zeta_{d m, d}\right)_{m \geqslant 1}$ is an i.i.d. sequence and the distribution of $\left(\zeta_{n, k}\right)_{k \geqslant 1}$ does not depend on $n$.

A key observation is that if we run the process up to time $k$, next we put all the particles at the position of $\zeta_{k}[N]$, and finally we run it for another $l$ steps, we obtain a configuration that dominates the one obtained by running the process $k+l$ steps. In other words,

$$
\zeta_{0, n+k}[N] \leqslant\left\{\zeta_{n, k}+\zeta_{0, n}[N]\right\}[N]
$$

Now, define $\gamma_{n, k}=\zeta_{n, k-n}$ for $0 \leqslant n \leqslant k$. Taking into account the aforementioned considerations it is simple to verify that the sequence $\left(\gamma_{n, k}[N]\right)_{n, k \geqslant 1}$ fulfills all the conditions of the subadditive ergodic theorem. Thus, $\lim _{k \rightarrow \infty} \gamma_{0, k}[N] / k$ exists almost surely and in $L^{1}$, and it is nonrandom. Finally, observe that $\left(\gamma_{0, k}\right)_{k \geqslant 0}$ and $\left(\zeta_{k}\right)_{k \geqslant 0}$ coincide in law and hence the result holds also for $\lim _{k \rightarrow \infty} \zeta_{k}[N] / k$ and $\lim _{t \rightarrow \infty} \xi_{t}[N] / t$. The last limit holds since $\left(\zeta_{k}[N]\right)_{k \geqslant 0}$ and $\left(\xi_{\tau_{k}}[N]\right)_{k \geqslant 0}$ have the same distribution and the maximum displacements of $\left(\xi_{t}[N]\right)_{t \geqslant 0}$ between jump times are tight (and hence converge to zero when divided by $t$ ). The existence of the $\operatorname{limit}_{t \rightarrow \infty} \xi_{t}[1] / t$ can be obtained in a similar way.

Finally observe that (1) and (2) of Theorem 2.3, imply that $\eta_{t}(N-1)$ converges in distribution, as $t \rightarrow \infty$ to an almost surely finite random variable $X$. Therefore, $\eta_{t}(N-1) / t=\left(\xi_{t}[N]-\xi_{t}[1]\right) / t$ converges in distribution to 0 and thus, also in probability. This finishes the proof of (3).

4.4. Velocity. To prove the monotonicity stated in (4) we use a construction similar to the one in (4.1) to couple two systems, one with $N$ particles, denoted by $\left(\zeta_{k}^{N}\right)_{k \geqslant 0}$, and another with $N+1$ particles denoted by $\left(\zeta_{k}^{N+1}\right)_{k \geqslant 0}$. This coupling is similar to the one used in Bérard and Gouéré (2010); Maillard (2016); Durrett and Remenik (2011) to prove the same monotonicity result. The main difference is that in those 
models the rate at which each pair of particles produce a branching-selection event is independent of $N$, while in our case it is $1 /(N-1)$.

For the ease of the reading, we first describe the coupling informally. Let us suppose that the initial conditions are ordered in the following sense

$$
\zeta_{0}^{N+1}[i+1] \geqslant \zeta_{0}^{N}[i], \quad i=1, \ldots, N .
$$

We start by matching the order statistics of both systems. Each $\zeta^{N}[i], i=1, \ldots, N$ is paired with $\zeta^{N+1}[i+1]$. The particle at $\zeta^{N+1}[1]$ is not coupled. We re-match the particles of the two processes just before and immediately after every time a jump takes place. We do not re-match between jumps, but during these periods the Brownian displacements are coupled among both systems according to the match. More precisely, after each jump we use the same Brownian motion to drive the particles at positions $\zeta^{N+1}[i+1]$ and $\zeta^{N}[i]$, for every $1 \leqslant i \leqslant N$. This guarantees that the pairs matched by the coupling keep their relative order. In particular, each $i$-th order statistic of the $N$-system has at least $N-i$ particles of the $(N+1)$-system to the right.

Given that the rate at which each pair of order statistics produce a jump in a system with $N$ particles is $\frac{1}{N-1}$ (and hence depends on $N$ ), we couple the jumps as follows. Each time there is a jump from position $\zeta^{N}[i]$ to position $\zeta^{N}[j]$ with $i<j$, we enforce the same jump from $\zeta^{N+1}[i+1]$ to $\zeta^{N+1}[j+1]$ with probability $\frac{N-1}{N}$ while, with probability $\frac{1}{N}$, the jump occurs from $\zeta^{N+1}[1]$ to $\zeta^{N+1}[j+1]$. Note that in order to obtain the correct rates for the $(N+1)$-system, extra jumps from $\zeta^{N+1}[1]$ to the other particles should be included. Observe that while this procedure gives the correct rates, neither of these jumps alter the ordering (4.4).

We now provide a precise construction. Let $\left(T_{k}\right)_{k \geqslant 1}$ be a Poisson process with rate $\lambda^{N}+\lambda^{+}$, with $\lambda^{N}:=\frac{N}{2}$ and $\lambda^{+}=\frac{1}{2}$. Let $\left(V_{k}\right)_{k \geqslant 1}$, with $V \sim \mathcal{U}\left\{O_{N}\right\}$ as in (4.1). We decompose $B_{s}^{N+1}=\left(B_{s}^{1}, B_{s}^{N}\right)$ where $\left(B_{s}^{N}\right)_{s \geqslant 0}$ is a $N$-dimensional Brownian motion. Finally, let $\left(W_{k}\right)_{k \geqslant 1}$ and $\left(C_{k}\right)_{k \geqslant 1}$ be two Bernoulli processes with success probability $\frac{\lambda^{N}}{\lambda^{N}+\lambda^{+}}$and $\frac{N-1}{N}$ respectively, and $\left(V_{k}^{+}\right)_{k \geqslant 1}$ i.i.d. random variables probabilty mass function

$$
p_{V_{1}^{+}}(j)=\frac{2}{N} \frac{N-j+1}{N-1}, \quad j=2, \ldots, N .
$$

Given $\zeta_{0}^{N}, \zeta_{0}^{N+1}$ (and using the previously defined notations $\sigma$ and $\theta$ ), we construct both processes as follows:

$$
\begin{gathered}
\zeta_{k+1}^{N}= \begin{cases}\sigma\left(\theta_{V_{k}}\left(\sigma\left(\zeta_{k}^{N}+B_{T_{k+1}}^{N}-B_{T_{k}}^{N}\right)\right)\right), & \text { if } W_{k}=1, \\
\zeta_{k}^{N}+B_{T_{k+1}}^{N}-B_{T_{k}}^{N}, & \text { if } W_{k}=0,\end{cases} \\
\zeta_{k+1}^{N+1}= \begin{cases}\sigma\left(\theta_{V_{k}+(1,1)}\left(\sigma\left(\zeta_{k}^{N+1}+B_{T_{k}+1}^{N+1}-B_{T_{k}}^{N+1}\right)\right)\right), & \text { if } W_{k}=1, C_{k}=1, \\
\sigma\left(\theta_{\left(1, V_{k}(2)+1\right)}\left(\sigma\left(\zeta_{k}^{N+1}+B_{T_{k+1}+1}^{N+1} B_{T_{k}}^{N+1}\right)\right)\right), & \text { if } W_{k}=1, C_{k}=0, \\
\sigma\left(\theta_{(1, j)}\left(\sigma\left(\zeta_{k}^{N+1}+B_{T_{k+1}}^{N+1}-B_{T_{k}}^{N+1}\right)\right)\right), & \text { if } W_{k}=0, V_{k}^{+}=j, j \geqslant 2 .\end{cases}
\end{gathered}
$$

It is easy to check that with this construction

$$
\zeta_{k}^{N+1}[i+1] \geqslant \zeta_{k}^{N}[i], \forall k \geqslant 1, \forall i=1, \ldots, N,
$$

which implies the claimed monotonicity $v_{N+1} \geqslant v_{N}$.

To prove (2.6) we provide a lower bound for the speed of the empirical mean and an upper bound for the speed of the rightmost particle. The result will follow from 
the fact that both bounds coincide as $N \rightarrow \infty$. For the lower bound, we make use of the monotonicity of the spacings of $\xi_{t}$, which is established in the next lemma.

Lemma 4.1. Let $\left(\xi_{t}\right)_{t \geqslant 0},\left(\tilde{\xi}_{t}\right)_{t \geqslant 0}$ be two processes with generator (2.1) and initial conditions $\xi_{0}, \tilde{\xi}_{0}$ respectively such that

$$
\xi_{0}[i+1]-\xi_{0}[i] \leqslant s t \tilde{\xi}_{0}[i+1]-\tilde{\xi}_{0}[i], \quad i=1, \ldots, N-1 .
$$

Then,

$$
\xi_{t}[i+1]-\xi_{t}[i] \leqslant s t \tilde{\xi}_{t}[i+1]-\tilde{\xi}_{t}[i], \quad i=1, \ldots, N-1, \quad \forall t \geqslant 0 .
$$

Here $\leqslant s t$ denotes stochastic domination.

Proof: Let us first observe that the result holds for the spacings of $N$ independent Brownian motions (see Ramasubramanian, 2000, Theorem 4.1), which is the law of the particles between jump times. Next, note that if the jumps for both processes are coupled as in (4.1) the domination of spacings is preserved after each jump. Proceeding inductively we obtain the stochastic domination for all times.

Lower bound. Let $u^{0}$ be the solution of the F-KPP equation with initial condition given by the Heavyside function and $w_{\sqrt{2}}(x)$ the minimal velocity traveling wave for (1.1). Let $m(t)$ be the median of $u^{0}(\cdot, t)$. The following facts hold (Bramson, 1983).

(i) $\left\|u^{0}(\cdot+m(t), t)-w_{\sqrt{2}}\right\| \rightarrow 0$ as $t \rightarrow \infty$.

(ii) $\sqrt{2}=\int_{-\infty}^{+\infty} w_{\sqrt{2}}(x)\left(1-w_{\sqrt{2}}(x)\right) d x$.

Our strategy consists in comparing the velocity of the empirical mean of the system in equilibrium as seen from the leftmost particle and the system with initial condition given by the Heavyside function. Afterwards, Theorem 2.1 will provide us the link to use (i)-(ii). This idea appears in Bramson et al. (1986).

We denote by $\bar{\nu}^{N}$ the distribution obtained by placing particle labeled 1 at the origin and the remaining ones according to $\nu^{N}$. Let $m_{t}^{N}=\frac{1}{N} \sum_{i=1}^{N} \xi_{t}(i)$ be the empirical mean and observe that

$$
E_{\bar{\nu}^{N}}\left[m_{t}^{N}\right]=\int_{0}^{+\infty} E_{\bar{\nu}^{N}}\left[1-F_{N}(x, t)\right] d x-\int_{-\infty}^{0} E_{\bar{\nu}^{N}}\left[F_{N}(x, t)\right] d x
$$

and hence differentiating under the integral sign we get

$$
\begin{aligned}
\partial_{t} E_{\bar{\nu}^{N}}\left[m_{t}^{N}\right] & =-\int_{-\infty}^{+\infty} \partial_{t} E_{\bar{\nu}^{N}}\left[F_{N}(x, t)\right] d x \\
& =\frac{N}{N-1} \int_{-\infty}^{+\infty} E_{\bar{\nu}^{N}}\left[F_{N}(x, t)\left(1-F_{N}(x, t)\right)\right] d x .
\end{aligned}
$$

In the last equality we make use of (3.6). Let us notice that

$$
F_{N}(x, t)=\mathbf{1}\left\{x \geqslant \xi_{t}[N]\right\}+\sum_{k=1}^{N-1} \frac{k}{N} \mathbf{1}\left\{\xi_{t}[k] \leqslant x<\xi_{t}[k+1]\right\},
$$

which gives us

$$
\int_{-\infty}^{+\infty} F_{N}(x, t)\left(1-F_{N}(x, t)\right) d x=\sum_{k=1}^{N-1} \frac{k(N-k)}{N^{2}}\left(\xi_{t}[k+1]-\xi_{t}[k]\right),
$$


and hence it is monotone in the spacings of the system.

We bound (4.5) from below as follows

$$
\begin{aligned}
\int_{-\infty}^{+\infty} E_{\bar{\nu}^{N}}\left[F_{N}(x, t)\left(1-F_{N}(x, t)\right)\right] d x & \geqslant \int_{-\infty}^{+\infty} E_{0}\left[F_{N}(x, t)\left(1-F_{N}(x, t)\right)\right] d x \\
& \geqslant \int_{m(t)-b}^{m(t)+b} E_{0}\left[F_{N}(x, t)\left(1-F_{N}(x, t)\right)\right] d x,
\end{aligned}
$$

for all $0<b<\infty$, where $E_{0}$ stands for the expectation with respect to the process distribution with initial configuration given by placing all the particles at the origin. Now, (4.6) can be written as

$$
\begin{aligned}
& \int_{m(t)-b}^{m(t)+b} E_{0}\left[F_{N}(x, t)\left(1-F_{N}(x, t)\right)\right] d x-\int_{m(t)-b}^{m(t)+b} u^{0}\left(1-u^{0}\right)(x, t) d x \\
+ & \int_{m(t)-b}^{m(t)+b} u^{0}\left(1-u^{0}\right)(x, t) d x .
\end{aligned}
$$

Due to (i)-(ii) we can fix $b$ and $t$ big enough to make (4.8) as close to $\sqrt{2}$ as desired. For those values of $b$ and $t$, the term (4.7) converges to zero as $N \rightarrow \infty$. Here we use that, due to Theorem 2.1, $\left\|F_{N}\left(1-F_{N}\right)(\cdot, t)-u^{0}\left(1-u^{0}\right)(\cdot, t)\right\|$ converges to zero in probability (under $P_{0}$ ) as $N \rightarrow \infty$; and hence it does it in $L^{1}$. So,

$$
\liminf _{N \rightarrow \infty} \int_{-\infty}^{+\infty} E_{\bar{\nu}^{N}}\left[F_{N}(x, t)\left(1-F_{N}(x, t)\right)\right] d x \geqslant \sqrt{2} .
$$

We claim that $\partial_{t} E_{\bar{\nu}^{N}}\left[m_{t}^{N}\right]=v_{N}$. Indeed, as $\nu^{N}$ is the equilibrium distribution for the system seen from the leftmost particle, a renewal argument readily implies that $\left(\xi_{t}[1]\right)_{t \geqslant 0}$ has stationary increments and thus

$$
E_{\bar{\nu}^{N}}\left[m_{t}^{N}\right]=E_{\bar{\nu}^{N}}\left[m_{t}^{N}-\xi_{t}[1]\right]+E_{\bar{\nu}^{N}}\left[\xi_{t}[1]\right]=c_{1}+c_{2} t,
$$

with $c_{1}$ and $c_{2}$ depending on $N$. The limit in (2.5) forces $c_{2}$ to equal $v_{N}$ which implies our claim. Combining this with (4.5) and (4.9) we conclude that

$$
\liminf _{n \rightarrow \infty} v_{N} \geqslant \sqrt{2} \text {. }
$$

Upper bound. For this bound, we embed the process into $N$ independent BBMs. Recall that the maximum $M_{t}$ of a BBM at time $t$ verifies

$$
\lim _{t \rightarrow \infty} \frac{M_{t}}{t}=\sqrt{2} \text { a.s. }
$$

We provide a last alternative construction of the process. Instead of using a Poisson clock of rate 1 for the jumps of each particle, we use a Poisson clock of rate $\frac{k-1}{N-1}$ for each $\operatorname{rank} k=1, \ldots, N$. Whenever the $k$-th clock rings the particle whose rank is $k$ branches into two particles. At that time, a particle is chosen uniformly at random among those particles with rank less than $k$ and is eliminated from the system. Between jumps, particles evolve as independent Brownian motions. It is clear that (i) the process constructed in this way has generator (2.1) and (ii) each particle branches at a rate which is less or equal than one. Hence we can couple this process with a BBM by adding extra branchings to achive rate one.

More precisely, let us construct a coupling between a process with generator (2.1) and $N$ independent BBM systems. We will still denote it $\left(\xi_{t}\right)_{t \geqslant 0}$. Let 
$X_{1}(t), \ldots, X_{N}(t), X_{N+1}(t), \ldots$ denote the positions of all the particles of $N$ independent BBM starting at $\xi_{0}(1), \ldots, \xi_{0}(N)$, labeled according to its appearence time. Let $\left(\tau_{i}\right)_{i \geqslant 1}$ be the branching times (in increasing order) and $\left(l_{i}\right)_{i \geqslant 1}$ the corresponding labels of the branching particles. Let $\left(U_{i}\right)_{i \geqslant 1}$ and $\left(D_{i}^{k}\right)_{k \leqslant N, i \geqslant 1}$ be independent random variables with $U_{i}$ uniform in [0,1], $i \geqslant 1$ and $D_{i}^{k}$ uniform in $\{1, \ldots, k-1\}$ for $i \geqslant 1, k \leqslant N$. Finally, let $\alpha_{k}=\frac{k-1}{N-1}, k=2, \ldots, N$. We construct a process $\left(L_{t}^{1}, \ldots, L_{t}^{N}\right)$ on the set of labels $\mathbb{N}$. We will use these labels to determine the particles of the BBM that are going to be used to construct the coupling. For $j=1, \ldots, N$, we define

$$
R_{t}^{j}=\operatorname{rank} \text { of } X_{L_{t}^{j}}(t) \text { in the set }\left\{X_{L_{t}^{1}}(t), \ldots, X_{L_{t}^{N}}(t)\right\} \subseteq\{1, \ldots, N\} .
$$

We build $\left(L_{t}^{1}, \ldots, L_{t}^{N}\right)$ inductively as follows:

- At time zero $\left(L_{0}^{1}, \ldots, L_{0}^{N}\right)=(1, \ldots, N)$.

- Assume $\left(L_{\tau_{i-1}}^{1}, \ldots, L_{\tau_{i-1}}^{N}\right)$ is known. For $t \in\left(\tau_{i-1}, \tau_{i}\right]$ we define:

$$
\begin{aligned}
L_{t}^{k} & = \begin{cases}N+i & \text { if } t=\tau_{i}, \quad l_{i}=L_{t^{-}}^{j}, j \neq k, \quad U_{i}<\alpha_{R_{t}^{j}}, \quad D_{i}^{R_{t}^{j}}=R_{t}^{k} \\
L_{t^{-}}^{k} & \text { otherwise },\end{cases} \\
k & =1, \ldots, N .
\end{aligned}
$$

We leave to the reader to verify that

$$
\left(\xi_{t}(1), \ldots, \xi_{t}(N)\right):=\left(X_{L_{t}^{1}}(t), \ldots, X_{L_{t}^{N}}(t)\right), \quad t \geqslant 0
$$

has generator 2.1. With this coupling we have that

$$
\xi_{t}[N] \leqslant \max \left(M_{t}^{1}, \ldots, M_{t}^{N}\right), \quad \forall t \geqslant 0,
$$

with $M_{t}^{j}$ the maximum of the $j$-th BBM. Combining (4.10) with (4.11) we conclude

$$
\limsup _{t \rightarrow \infty} \frac{\xi_{t}[N]}{t} \leqslant \sqrt{2}
$$

\section{Acknowledgments}

We thank Nahuel Soprano-Loto for pointing out an error in a preliminary version of the manuscript and the anonymous reviewer for a careful reading of the manuscript and several comments that helped us to improve the article. The authors are partially supported by grants UBACYT 20020160100147BA and PICT 2015-3154.

\section{References}

Asmussen, S. Applied probability and queues, volume 51 of Applications of Mathematics (New York). Springer-Verlag, New York, second edition (2003). ISBN 0-387-00211-1. MR1978607.

Asselah, A., Ferrari, P. A., and Groisman, P. Quasistationary distributions and Fleming-Viot processes in finite spaces. J. Appl. Probab., 48 (2), 322-332 (2011). MR2840302.

Asselah, A., Ferrari, P. A., Groisman, P., and Jonckheere, M. Fleming-Viot selects the minimal quasi-stationary distribution: the Galton-Watson case. Ann. Inst. Henri Poincaré Probab. Stat., 52 (2), 647-668 (2016). MR3498004.

Athreya, K. B. and Ney, P. A new approach to the limit theory of recurrent Markov chains. Trans. Amer. Math. Soc., 245, 493-501 (1978). MR511425. 
Benguria, R. and Depassier, M. Speed of pulled fronts with a cutoff. Physical Review E, 75 (5), 051106 (2007). DOI: 10.1103/PhysRevE.75.051106.

Bérard, J. and Gouéré, J.-B. Brunet-Derrida behavior of branching-selection particle systems on the line. Comm. Math. Phys., 298 (2), 323-342 (2010). MR2669438.

Berestycki, J., Brunet, E., and Derrida, B. Exact solution and precise asymptotics of a Fisher-KPP type front. J. Phys. A, 51 (3), 035204, 21 (2018). MR3741997.

Berestycki, J., Brunet, E., and Penington, S. Global existence for a free boundary problem of Fisher-KPP type. Nonlinearity, 32 (10), 3912-3939 (2019). MR4012576.

Billingsley, P. Convergence of probability measures. Wiley Series in Probability and Statistics: Probability and Statistics. John Wiley \& Sons, Inc., New York, second edition (1999). ISBN 0-471-19745-9. MR1700749.

Bramson, M. Convergence of solutions of the Kolmogorov equation to travelling waves. Mem. Amer. Math. Soc., 44 (285), iv+190 (1983). MR705746.

Bramson, M., Calderoni, P., De Masi, A., Ferrari, P., Lebowitz, J., and Schonmann, R. H. Microscopic selection principle for a diffusion-reaction equation. J. Statist. Phys., 45 (5-6), 905-920 (1986). MR881315.

Brunet, E. and Derrida, B. Shift in the velocity of a front due to a cutoff. Phys. Rev. E (3), 56 (3, part A), 2597-2604 (1997). MR1473413.

Brunet, E. and Derrida, B. Effect of microscopic noise on front propagation. $J$. Statist. Phys., 103 (1-2), 269-282 (2001). MR1828730.

Brunet, E., Derrida, B., Mueller, A. H., and Munier, S. Noisy traveling waves: effect of selection on genealogies. Europhys. Lett., 76 (1), 1-7 (2006). MR2299937.

Brunet, E., Derrida, B., Mueller, A. H., and Munier, S. Effect of selection on ancestry: an exactly soluble case and its phenomenological generalization. Phys. Rev. E (3), 76 (4), 041104, 20 (2007). MR2365627.

De Masi, A., Ferrari, P. A., Presutti, E., and Soprano-Loto, N. Hydrodynamics of the $N$-BBM process. In Stochastic dynamics out of equilibrium, volume 282 of Springer Proc. Math. Stat., pp. 523-549. Springer, Cham (2019). MR3986078.

Dumortier, F., Popović, N., and Kaper, T. J. The critical wave speed for the FisherKolmogorov-Petrowskii-Piscounov equation with cut-off. Nonlinearity, 20 (4), 855-877 (2007). MR2307884.

Durrett, R. Probability. Theory and examples. The Wadsworth \& Brooks/Cole Statistics/Probability Series. Wadsworth \& Brooks/Cole Advanced Books \& Software, Pacific Grove, CA (1991). ISBN 0-534-13206-5. MR1068527.

Durrett, R. and Remenik, D. Brunet-Derrida particle systems, free boundary problems and Wiener-Hopf equations. Ann. Probab., 39 (6), 2043-2078 (2011). MR2932664.

Fisher, R. The wave of advance of advantageous genes. Ann. Eugenics, 7, 353-369 (1937).

Groisman, P. and Jonckheere, M. Front Propagation and Quasi-Stationary Distributions: Two Faces of the Same Coin. In Sidoravicius, V., editor, Sojourns in Probability Theory and Statistical Physics - III, pp. 242-254. Springer Singapore, Singapore (2019). ISBN 978-981-15-0302-3. DOI: 10.1007/978-981-15-0302-3 9.

Groisman, P. and Soprano-Loto, N. A family of microscopic models in the F-KPי class. In preparation (2020). 
Kallenberg, O. Foundations of modern probability. Probability and its Applications (New York). Springer-Verlag, New York, second edition (2002). ISBN 0-38795313-2. MR1876169.

Kolmogorov, A., Petrovsky, I., and Piscounov, N. Etude de l'equation de la diffusion avec croissance de la quantite de matiere et son application a un probleme biologique. Bull. Univ. Etat Moscou, A 1:1 (25) (1937).

Lyons, R. Strong laws of large numbers for weakly correlated random variables. Michigan Math. J., 35 (3), 353-359 (1988). MR978305.

Maillard, P. Speed and fluctuations of $N$-particle branching Brownian motion with spatial selection. Probab. Theory Related Fields, 166 (3-4), 1061-1173 (2016). MR3568046.

McKean, H. P. Application of Brownian motion to the equation of KolmogorovPetrovskii-Piskunov. Comm. Pure Appl. Math., 28 (3), 323-331 (1975). MR400428.

Mueller, C., Mytnik, L., and Quastel, J. Small noise asymptotics of traveling waves. Markov Process. Related Fields, 14 (3), 333-342 (2008). MR2453698.

Mueller, C., Mytnik, L., and Quastel, J. Effect of noise on front propagation in reaction-diffusion equations of KPP type. Invent. Math., 184 (2), 405-453 (2011). MR2793860.

Quittner, P. and Souplet, P. Superlinear parabolic problems. Blow-up, global existence and steady states. Birkhäuser Advanced Texts: Basler Lehrbücher. [Birkhäuser Advanced Texts: Basel Textbooks]. Birkhäuser Verlag, Basel (2007). ISBN 978-3-7643-8441-8. MR2346798.

Ramasubramanian, S. A subsidy-surplus model and the Skorokhod problem in an orthant. Math. Oper. Res., 25 (3), 509-538 (2000). MR1855180.

Roelly-Coppoletta, S. A criterion of convergence of measure-valued processes: application to measure branching processes. Stochastics, 17 (1-2), 43-65 (1986). MR878553. 